\title{
Video Article \\ Isolation of Human Endothelial Cells from Normal Colon and Colorectal Carcinoma - An Improved Protocol
}

\author{
Elisabeth Naschberger ${ }^{1}$, Daniela Regensburger ${ }^{1}$, Clara Tenkerian ${ }^{1}$, Melanie Langheinrich ${ }^{2}$, Felix B. Engel ${ }^{3}$, Carol Geppert ${ }^{4}$, Arndt Hartmann $^{4}$, \\ Robert Grützmann ${ }^{2}$, Vera S. Schellerer ${ }^{2}$, Michael Stürzl ${ }^{* 1}$ \\ ${ }^{1}$ Division of Molecular and Experimental Surgery, Department of Surgery, University Medical Center Erlangen, Friedrich-Alexander University of Erlangen-Nuremberg \\ ${ }^{2}$ Department of Surgery, University Medical Center Erlangen, Friedrich-Alexander University of Erlangen-Nuremberg \\ ${ }^{3}$ Division of Nephropathology, Department of Pathology, University Medical Center Erlangen, Friedrich-Alexander University of Erlangen-Nuremberg \\ ${ }^{4}$ Department of Pathology, University Medical Center Erlangen, Friedrich-Alexander University of Erlangen-Nuremberg \\ "These authors contributed equally
}

Correspondence to: Michael Stürzl at Michael.Stuerzl@uk-erlangen.de

URL: https://www.jove.com/video/57400

DOI: doi:10.3791/57400

Keywords: Cancer Research, Issue 134, Endothelial Cells, Vessel, Colorectal Carcinoma, Tumor, Normal Colon, CD31, VE-cadherin, CD105, FACS-sorting, Tissue Dissociation, Tumor Microenvironment, Fibroblasts

Date Published: 4/4/2018

Citation: Naschberger, E., Regensburger, D., Tenkerian, C., Langheinrich, M., Engel, F.B., Geppert, C., Hartmann, A., Grützmann, R., Schellerer, V.S., Stürzl, M. Isolation of Human Endothelial Cells from Normal Colon and Colorectal Carcinoma - An Improved Protocol. J. Vis. Exp. (134), e57400, doi:10.3791/57400 (2018).

\section{Abstract}

Primary cells isolated from human carcinomas are valuable tools to identify pathogenic mechanisms contributing to disease development and progression. In particular, endothelial cells (EC) constituting the inner surface of vessels, directly participate in oxygen delivery, nutrient supply, and removal of waste products to and from tumors, and are thereby prominently involved in the constitution of the tumor microenvironment (TME). Tumor endothelial cells (TECs) can be used as cellular biosensors of the intratumoral microenvironment established by communication between tumor and stromal cells. TECs also serve as targets of therapy. Accordingly, in culture these cells allow studies on mechanisms of response or resistance to anti-angiogenic treatment. Recently, it was found that TECs isolated from human colorectal carcinoma (CRC) exhibit memory-like effects based on the specific TME they were derived from. Moreover, these TECs actively contribute to the establishment of a specific TME by the secretion of different factors. For example, TECs in a prognostically favorable Th1-TME secrete the anti-angiogenic tumorsuppressive factor secreted protein, acidic and rich in cysteine-like 1 (SPARCL1). SPARCL1 regulates vessel homeostasis and inhibits tumor cell proliferation and migration. Hence, cultures of pure, viable TECs isolated from human solid tumors are a valuable tool for functional studies on the role of the vascular system in tumorigenesis. Here, a new up-to-date protocol for the isolation of primary EC from the normal colon as well as CRC is described. The technique is based on mechanical and enzymatic tissue digestion, immunolabeling, and fluorescence activated cell sorting (FACS)-sorting of triple-positive cells (CD31, VE-cadherin, CD105). With this protocol, viable TEC or normal endothelial cell (NEC) cultures could be isolated from colon tissues with a success rate of $62.12 \%$ when subjected to FACS-sorting (41 pure EC cultures from 66 tissue samples). Accordingly, this protocol provides a robust approach to isolate human EC cultures from normal colon and CRC.

\section{Video Link}

The video component of this article can be found at https://www.jove.com/video/57400/

\section{Introduction}

The tumor microenvironment (TME) is defined as a close interaction of tumor cells with the tumor stroma, which is comprised of cells such as endothelial cells $(E C)$, pericytes, fibroblasts, smooth muscle cells, or immune cells. The communication between these cellular compartments can be driven by paracrine factors (e.g., angiogenic growth factors, cytokines), by the extracellular matrix, or direct cell-cell contact. The stromal compartment may foster or counteract tumor initiation or progression, depending on the specific TME established.

The ability of a tumor to connect with the vessel system is key to the progression and metastasis of the disease. The vessel system allows the tumor predominantly to gain access to the delivery of oxygen and nutrients as well as the removal of waste products ${ }^{1,2,3}$. EC constitute the inner surface of vessels, and are therefore important cellular components that actively participate in this process. It is well known that tumor endothelial cells (TEC) are different to their corresponding normal endothelial cells (NEC) by many features such as disturbed hierarchy of the vascular tree, vessel leakiness, or reduced maturation as exemplified by a reduced number of pericytes/mural cells that are only loosely attached to the $\mathrm{EC}^{4}$.

Hence, TECs are valuable cellular tools to study carcinogenesis. TECs were primarily considered to foster tumor growth and progression ${ }^{3}$. Thus, TECs can be used as biosensors that allow the monitoring and identification of pathogenic processes that initiate, foster, or counteract 
tumorigenesis. Moreover, they are therapeutic targets in the clinic ${ }^{5}$. Consequently, isolated TECs and corresponding NECs may also be used as tools to understand mechanisms of response or resistance to anti-angiogenic treatment.

In the past, we developed a protocol to isolate these cells $s^{6,7}$ and identified that TECs are not only different from NECs, but also differ from each other depending on the TME they were derived from ${ }^{8}$. Through this approach, it was shown that TECs in certain TMEs can actively counteract tumor growth and progression by secretion of anti-angiogenic tumor-suppressive proteins such as SPARCL1. This indicated that TECs are actively contributing to the establishment of a prognostically favorable TME in human colorectal carcinoma (CRC).

Previous studies attempted to isolate human TECs from solid tumors. An important goal of these studies was, e.g., the identification of new tumor endothelial cell markers (TEMs) ${ }^{9}$. A strategy of immediate use of TECs after laser microdissection was applied in order to avoid alteration or loss of the TEC phenotype in culture. However, follow-up studies identified a contaminating population of mural cells as a serious drawback of this approach ${ }^{10}$. Our lab was the first to develop a protocol that allowed the isolation of pure, viable TECs from human CRC patients ${ }^{6,7}$. An approach with multiple magnetic cell selection (MACS) rounds of the TECs that ensured high purity of the isolated EC cultures was chosen. However, this approach required a relatively long cultivation period (6 weeks on average), which increased the risk of culture-induced artefacts. Hence, in the next step, the aim was to reduce the cultivation time between surgery and harvest of the first pure culture. To achieve this goal, an improved protocol employing a combined mechanical and enzymatic-based tissue dissociation of the initial tumor, followed by fluorescence activated cell sorting (FACS)-sorting of triple-labeled EC, was developed. This reduced isolation time on average to three weeks, resulting in pure TEC and NEC cultures with increased viability for functional studies. Isolation of pure viable TEC and NEC cultures from human tissues with high success rates may open new avenues for patient-specific drug testing during the development of individualized therapy regimens. The isolation approach is detailed in the following paragraphs.

\section{Protocol}

The isolation process has been approved by the local ethics committee of the University Medical Center Erlangen (\#159_15 B, TuMiC-study). Patient inclusion criteria were as follows: CRC, UICC stage I-IV, no history of inflammatory bowel disease, and no neoadjuvant treatment.

\section{Surgery and Preparation of Tissue for Single Cell Isolation}

1. Obtain specimen by surgery from a CRC patient (carcinoma tissue $>0.5 \mathrm{~g}$, central non-necrotic tumor; normal colon tissue $>10 \mathrm{~cm}$ distant from tumor site).

2. The obtained tissue pieces are mostly $<1 \mathrm{~g}$ for the carcinoma, but $>1 \mathrm{~g}$ for the normal colon. If pieces $>1 \mathrm{~g}$ are obtained, split them into multiple $1 \mathrm{~g}$ pieces using a fresh scalpel for further tissue processing.

NOTE: If the tumor tissue piece is below $0.5 \mathrm{~g}$ from the onset, the isolation usually fails.

3. Collect fresh unfixed tissue pieces using sterile forceps in $40 \mathrm{~mL}$ of ice cold Hanks balanced salt solution (HBSS) supplemented with penicillin/streptomycin/amphothericin B (1\% HBSS-pen/strep/ampho) in 50-mL centrifuge tubes and transfer them quickly to the laboratory. NOTE: Colon tissue is frequently highly contaminated with bacteria/fungi from the onset. During the whole procedure of isolation, pen/strep/ ampho must be added to all solutions in order to eliminate contaminating organisms.

4. Wash each tissue piece at least 4 times with $40 \mathrm{~mL}$ ice cold HBSS-pen/strep/ampho (see step 1.3) in 50 -mL centrifuge tubes by transferring the tissue pieces sequentially from one centrifuge tube to the next tube using sterile forceps.

1. Clean forceps after each step with $70 \%$ ethanol.

2. Use separate forceps for the carcinoma and normal colon tissue pieces.

3. Weigh all tissue pieces.

NOTE: Do not put tissue pieces directly on a scale for weighing. After washing, weigh the last centrifuge tube containing the tissue before and after entering the tissue pieces. Calculate the weight of individual tissue pieces by subtraction.

\section{Generation of Single Cell Suspension}

Note: It is recommended to keep the tissue moisturized at all times.

1. If it exists, remove attached fat, other non-tumorous tissue, or potentially necrotic tissue parts from the surgery specimen by transferring the tissue pieces in a sterile cell culture petri dish using sterile forceps.

1. Keep the tissue pieces moisturized at all times by adding 3-5 $\mathrm{mL}$ of HBSS-pen/strep/ampho.

2. Trim the tissue pieces using a fresh sterile scalpel (Figure 1). Consult a pathologist in case tissue identification is unclear.

2. Mince remaining tissue into small pieces (approximately $2 \times 2 \times 2 \mathrm{~mm}^{3}$ ) using a fresh scalpel with a curved blade. Transfer tissue pieces with sterile forceps into tissue dissociation tubes (for example, gentleMACS C tubes) pre-filled with pre-warmed $\left(37^{\circ} \mathrm{C}\right)$ cell culture medium (Dulbecco's modified eagle medium [DMEM] basal medium) according to the manufacturer's instructions.

NOTE: Try to use the scalpel like a rocking tool. Do not squeeze the cells in order to avoid tissue damage.

3. Digest/dissociate tissue pieces using a tissue dissociator (like gentleMACS Octo Dissociator) in combination with the tumor dissociation kit for human tissue using the program "37C_h_TDK_1" following the manufacturer's instructions.

4. Centrifuge dissociation tubes for $1 \mathrm{~min}$ at $300 \mathrm{xg}$ at room temperature (RT).

5. Add $10 \mathrm{~mL}$ of pre-warmed $\left(37^{\circ} \mathrm{C}\right)$ DMEM supplemented with $0.5 \%$ fetal bovine serum (FBS) (DMEM-Low) to each tube using a serological pipette and filter the cell suspension using a cell strainer ( $100 \mu \mathrm{m}$ pore size) on top of a $50-\mathrm{mL}$ centrifuge tube by pipetting the cell suspension on top of the filter.

6. Add $10 \mathrm{~mL}$ of DMEM-Low again to each MACS-tube by a serological pipette and transfer remaining cells to the cell strainer on top of the 50 $\mathrm{mL}$ centrifuge tube.

7. Wash the cell strainer once with an additional $10 \mathrm{~mL}$ of DMEM-Low medium using a serological pipette. 
8. Centrifuge the cell suspension for $7 \mathrm{~min}$ at $300 \mathrm{xg}$ at RT and discard supernatant.

9. Resuspend cells in $5 \mathrm{~mL}$ of pre-warmed endothelial basal medium (EBM)-2-microvascular (MV) medium (Lonza) supplemented with pen/ strep/ampho in the same $50-\mathrm{mL}$ centrifuge tube.

10. Plate cell suspension in a T-25 cell culture flask pre-coated overnight with $1.5 \%$ gelatin-phosphate buffered saline (PBS) at $37{ }^{\circ} \mathrm{C}$ with $5 \%$ $\mathrm{CO}_{2}$.

11. Incubate cells for $24 \mathrm{~h}$ at $37^{\circ} \mathrm{C}$ and $5 \% \mathrm{CO}_{2}$, wash the cells two times carefully with approximately $5 \mathrm{~mL}$ of PBS, and add $5 \mathrm{~mL}$ of fresh medium.

12. Cultivate the cells at $37{ }^{\circ} \mathrm{C}$ and $5 \% \mathrm{CO}_{2}$ until $80-90 \%$ confluency (usually $5-7$ days) with $48 \mathrm{~h}$ intervals of medium renewal.

\section{FACS-sorting of Endothelial Cells}

NOTE: Try to avoid losing cells at any point, e.g. by limiting the staining procedure to incubation of the cells in a single reagent tube.

1. Detach cells with $1 \mathrm{~mL}$ of accutase (approximately $10 \mathrm{~min}$ ), and add $4 \mathrm{~mL}$ of pre-warmed $\left(37^{\circ} \mathrm{C}\right)$ EBM-2-MV medium.

2. Determine cell count using an automated cell counting device (range $10-25 \mu \mathrm{m}$ ).

3. Centrifuge cell suspension for 4 min with $250 \times \mathrm{g}$ at RT and discard supernatant.

4. Resuspend cells in pre-warmed FACS-buffer (1x PBS, 2.5\% bovine serum albumin [BSA], $5 \mathrm{mM}$ EDTA pH 8.0, $10 \mu \mathrm{M}$ Y-27632) according to cell count $\left(5 \times 10^{6} / \mathrm{mL}\right)$.

5. Add FACS-staining antibodies according to cell count/FACS-buffer volume: CD31-FITC $(100 \mu \mathrm{L} / \mathrm{mL}=1 / 10)$, CD144/vascular endothelial (VE)-cadherin-PE $(100 \mu \mathrm{L} / \mathrm{mL}=1 / 10)$, CD105-APC $(25 \mu \mathrm{L} / \mathrm{mL}=1 / 40)$, mix and incubate for 7 min at RT protected from light; gently flick and incubate for another $8 \mathrm{~min}$ (for a total incubation time of $15 \mathrm{~min}$ ).

6. Add $2 \mathrm{~mL}$ of FACS-buffer to the labeled cell suspension using a serological pipette.

7. Centrifuge for 4 min with $250 \times \mathrm{g}$ at RT and discard supernatant.

8. Wash two times by adding $2 \mathrm{~mL}$ of FACS-buffer, and conduct a subsequent centrifugation for 4 min at $250 \times \mathrm{g}$ at RT and discard supernatant.

9. Resuspend cells in $500 \mu \mathrm{L}$ of pre-warmed EBM-2-MV-pen/strep/ampho and transfer cells to the FACS-sorting instrument.

1. Endothelial cells will quickly die during the sorting process if the FACS-instrument is cooled. Therefore, carry out sorting of endothelial cells at RT and immediately transfer the collected cells to the $37^{\circ} \mathrm{C}$ incubator afterwards.

NOTE: The FACS-instrument is usually unsterile!

10. Sort/collect triple-positive cells directly into a cell culture dish (e.g., 24-well plate pre-coated overnight with $1.5 \%$ gelatin-PBS) filled with 0.5 $\mathrm{mL}$ of pre-warmed fresh medium (EBM-2-MV-pen/strep/ampho).

NOTE: Check cell suspension after sorting using a microscope. If the cells cluster together, try to obtain an even cell distribution in the cell culture dish by mixing them slowly or adding medium.

11. Cultivate cells until $90-100 \%$ confluency with $48 \mathrm{~h}$ intervals of medium renewal and expand the cells to the desired culture dish size (24-well plate $\rightarrow 12$ well-plate $\rightarrow 3.5 \mathrm{~cm}$ dish $\rightarrow \mathrm{T}-25 \rightarrow \mathrm{T}-75$ ).

\section{Harvest and Characterization of Pure Endothelial Cells}

1. Harvest cells at $90-100 \%$ confluency and characterize them by an appropriate method (e.g., FACS, cytochemistry, quantitative polymerase chain reaction ( $\mathrm{qPCR}$ ), or endothelial cell function assays $\mathrm{s}^{7,8}$ ).

2. Analyze isolated cells with whichever characterization method is chosen.

\section{Representative Results}

The isolation of NEC and TEC from human CRC by a combined mechanical/enzymatical tissue dissociation, followed by subsequent CD31/ CD105/VE-cadherin-driven FACS-sorting is described here (Figure 1A). This protocol represents an improved protocol with a reduced time window until the first harvest of pure, viable endothelial cells as compared to the previous MACS-based protocol ${ }^{7}$.

NEC and TEC were isolated from human CRC using the following steps: (1) generation of a single cell suspension by combined mechanical and enzymatic tissue dissociation and growth of these cells to $80-90 \%$ confluency (Figure 1), (2) triple labeling of the EC by CD31/CD105/VEcadherin fluorochrome-coupled antibodies, and (3) FACS-sorting of the respective triple-positive cells (Figure 2A). Of note, a critical step for the success of the isolation procedure and viability of the cells is to quickly subject the surgery specimen to the isolation procedure (generation of single cell suspension $<1$ hour after resection). Using FACS-sorting, a mean of 12,500 TEC $(n=12)$ and $17,800 \mathrm{NEC}(\mathrm{n}=12$, Figure 2B, left) representing an average of 3.6 and $5.6 \%$ of the total cell population (Figure 2B, right) could be isolated. Subsequently, the cells were expanded up to T-75 (termed passage 1) and either harvested or further processed for analysis. Of note, using the previous MACS-based protocol, an average isolation success of $49.6 \%$ was achieved $\left(n=58\right.$ pure TECs from $n=117$ patients $\left.{ }^{8}\right)$ with pure EC cultures available on average 6 weeks after surgery. Using the new FACS-based protocol described here, the first pure EC cultures were obtained on average 3 weeks after surgery with an isolation success rate of $62.1 \%(n=41$ pure EC cultures obtained from $n=66$ single cells suspensions subjected to FACSsorting). Therefore, an increase of the isolation rate by $12.5 \%$ was achieved in parallel with a decrease of the cultivation time from 6 to 3 weeks. This reduction of the cultivation time led to improved cell viability and prolonged life span accompanied by less exposure to the induction of potential culture-dependent artefacts of the established cultures.

Characterization of EC purity was conducted first by CD31-cytochemistry (Figure 3A). If the cultures were devoid of CD31-negative cells (Figure 3A, TEC and NEC), they were subjected to a more detailed cell typing by reverse transcription quantitative polymerase chain reaction (RT-qPCR) (Figure 3B). Cell-type specific primers for EC (CD31, CD105, VE-cadherin, and von Willebrand factor [VWF]), leukocytes (CD45), epithelial cells (cytokeratin [CK]-20), and smooth muscle cells/fibroblasts (desmin) were used (Figure 3B). Using this qPCR-based cell typing, a potential contamination of the EC cultures by other cells in the range of $0.1-2 \%$, depending on the cell type, can be detected ${ }^{8}$. 
Notably, we previously reported a preferential loss of VWF protein expression in TEC cultures compared to their corresponding NEC cultures ${ }^{7}$. These findings could be confirmed with the newly established isolation protocol (Figure 3C, mean Ct loss NEC-TEC $=0.687, p=0.173$, paired ttest). Cultures successfully passing these quality controls were tested for mycoplasma infection and subsequently used for further experiments.

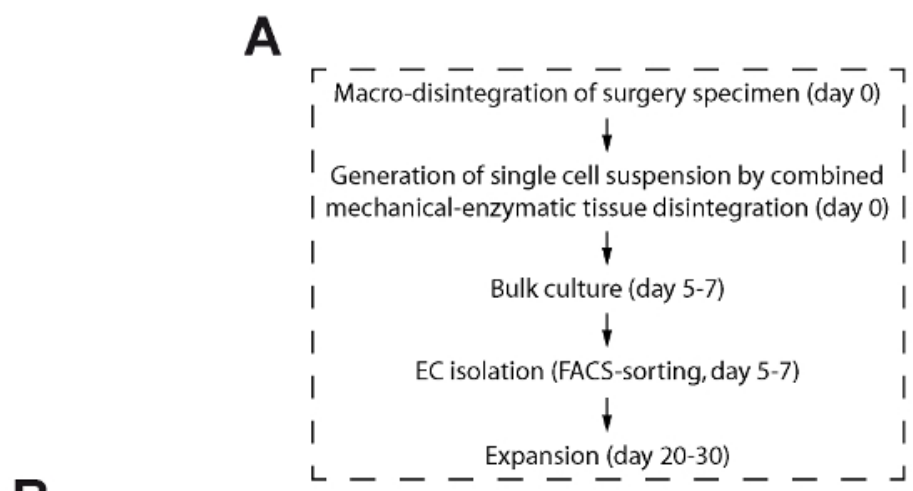

B
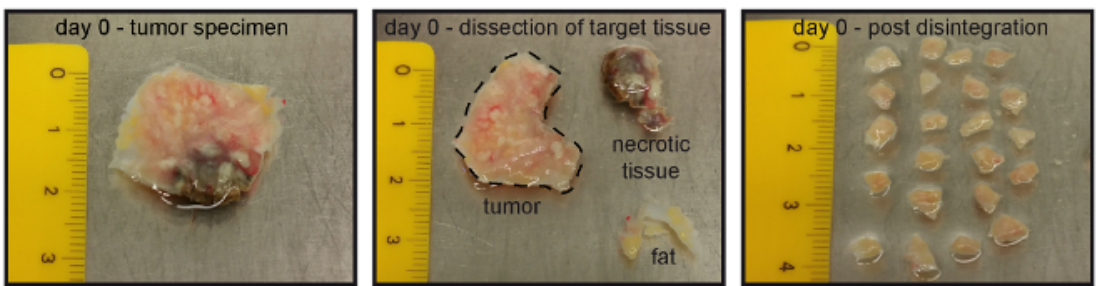

C
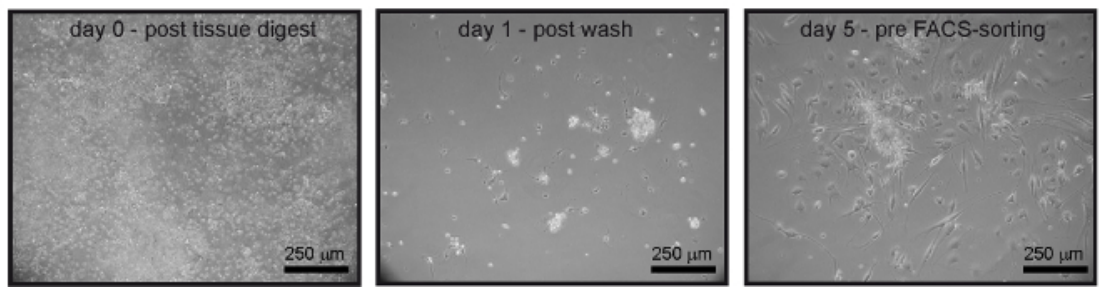

Figure 1: Pure endothelial cells can be isolated from human normal colon and CRC by FACS-sorting. (A) Flow chart of the essential steps of the EC isolation process. (B) Fat tissue (yellow), other non-tumorous parts, or potentially necrotic tissue parts (brown) were removed from the surgery specimen (left and middle panel) and the tumor was disintegrated into cubes of $2-3 \mathrm{~mm}$ side length prior to tissue dissociation (right panel). (C) The single cell suspension retrieved after tissue dissociation (left panel) was incubated for $24 \mathrm{~h}$ in cell culture dishes. Subsequently, non-adherent cells were removed by gentle washing using PBS (middle panel) and the cells were grown to 80 - $90 \%$ confluency prior to FACSsorting (right panel). Images of the lower panel $(C)$ were acquired by phase contrast microscopy. Please click here to view a larger version of this figure. 

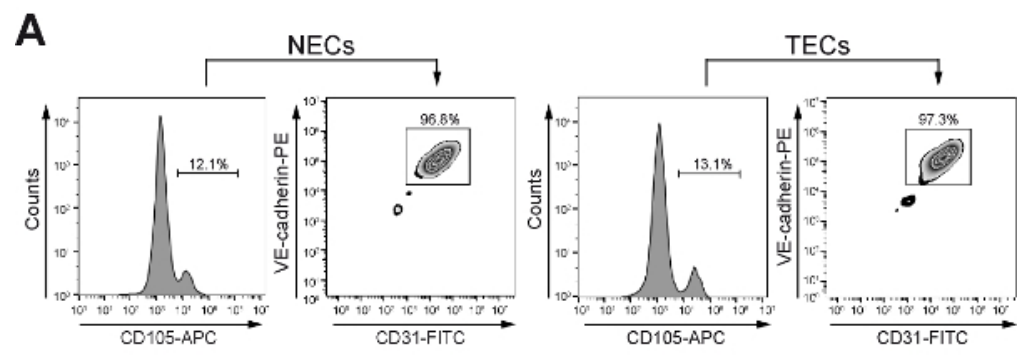

B
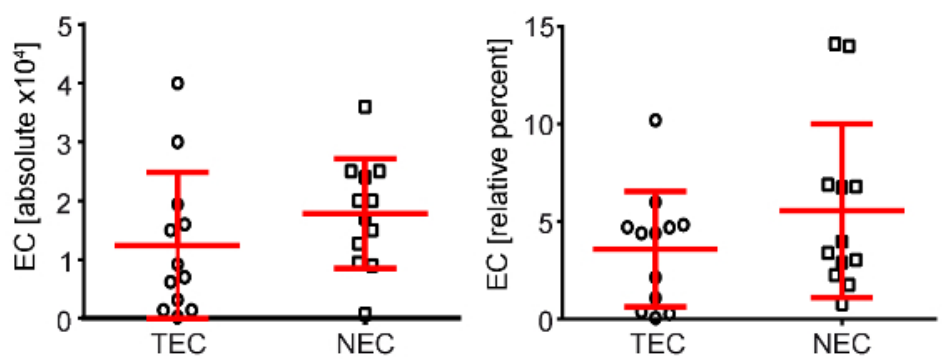

Figure 2: An average of $3.6-\mathbf{5 . 6 \%}$ of the single cell suspension isolated from human CRC patients and sorted by FACS were triplepositive EC. (A) NEC and TEC were FACS-sorted using a triple labeling of the cells with anti-CD31, -CD105 and -VE-cadherin antibodies. (B) A mean of 12,500 TECs $(n=12)$ and 17,800 NECs $(n=12)$ can be isolated from human normal colon or CRC using FACS-sorting (CD31, CD105 and VE-cadherin-positive cells, left panel), which represents on average 3.6 and $5.6 \%$ (right panel) of the total cell population employed. Please click here to view a larger version of this figure.

A
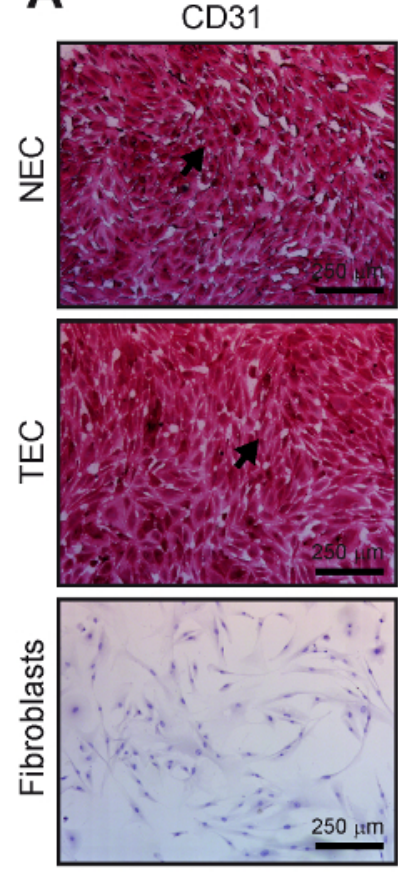

B

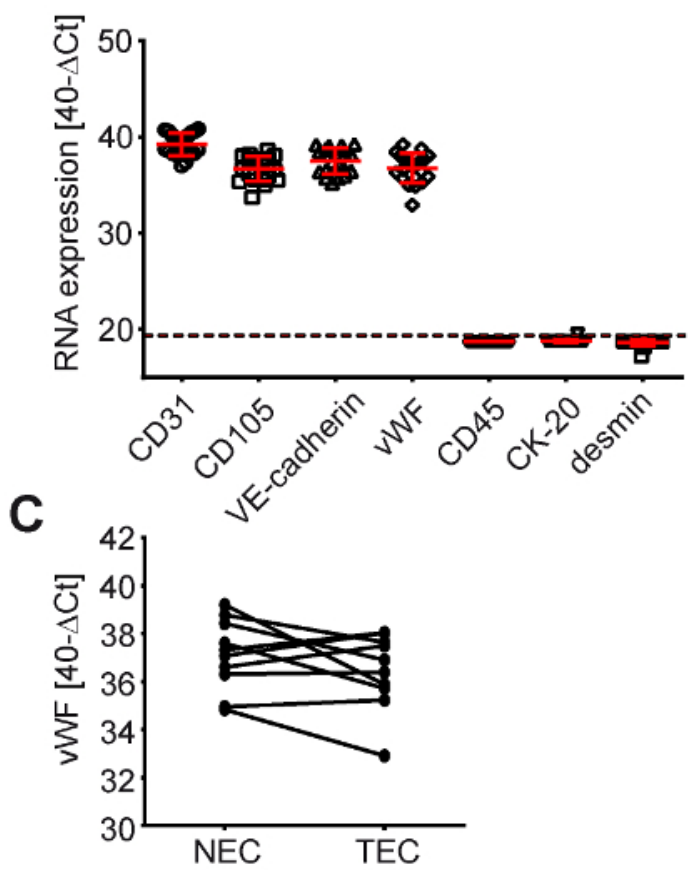

Figure 3: NEC and TEC from human CRC express typical endothelial cell markers. Normal colon endothelial cells $(N E C, n=10)$ and tumor endothelial cells (TEC, $n=10$ ) from human CRC are CD31 (EC marker)-, CD105 (EC marker)-, VE-cadherin (EC marker)-, vWF (EC marker)-positive and CK20 (CRC cell marker)-, desmin (fibroblast/SMC marker)-, CD45 (leukocyte marker)-negative as determined by (A) CD31immunocytochemistry (positive cells $=$ red) and $(\mathbf{B})$ RT-qPCR (data points below the dashed line are samples detected to be negative). (C) vWF expression as determined by RT-qPCR for corresponding TEC/NEC samples from the same patients. Please click here to view a larger version of this figure.

\section{Discussion}

Mainly, three different methods have been employed thus far to isolate pure and viable NECs and TECs from human solid tissues, namely (1) immunomagnetic enrichment, (2) laser microdissection, and (3) FACS-sorting of the EC fraction. In most publications, immunomagnetic 
enrichment of the cells after generation of a single cell suspension by mechanical disintegration was used ${ }^{11,12,13}$. For example, immunomagnetic purification of human dermal microvascular EC (HDMEC) by E-selectin antibodies after tumor necrosis factor (TNF)- $\alpha$ treatment from neonatal foreskins ${ }^{13}$ has been reported for the isolation of human EC from glioma by tissue digest, percoll gradient, and selection using anti-CD31/CD105 and VE-cadherin-antibodies ${ }^{12}$, or by anti-CD105 antibodies from human breast carcinoma ${ }^{11}$. Protocols that employed laser microdissection or FACS-sorting have been less frequently reported. Laser microdissection was used, for example, to identify potential pan-tumor endothelial cell markers (TEMs) in EC derived from human colorectal carcinoma with analysis of the cells immediately after dissection ${ }^{9}$. FACS-sorting using antiCD31-antibodies was successfully demonstrated for the isolation of the EC fraction from undifferentiated human embryonic stem cells ${ }^{14}$.

These approaches have different advantages and disadvantages that have to be considered. The advantages for the immunomagnetic-based approach are that no elaborate equipment is required, the selection can be conducted any time, and the cell enrichment is fast (approximately $15 \mathrm{~min}$ ) as compared to FACS-sorting (approximately $1 \mathrm{~h}$ ). The lack of matrix for a longer period of time may induce cell death of EC by anoikis Therefore, addition of the rho kinase inhibitor Y-27632 to the FACS buffer was employed to prevent cell anoikis ${ }^{15}$.

The disadvantages of immunomagnetic enrichment are that multiple rounds of selection are required in order to achieve purity above $99 \%$. Moreover, cell cultivation between enrichments is required for cell recovery, which increases the age of the cultures supporting culture-induced artefacts. Laser microdissection has the advantage that cells can be used immediately which reduces culture-induced artefacts but includes the risk of contamination by cells from adjacent tissue areas ${ }^{10}$. Moreover, microdissection is not established in every laboratory. The disadvantages of the FACS-sorting-based approach include, similar to laser microdissection, that the equipment is elaborate and cost-intensive. Accordingly, this equipment may not be accessible at any time. In a clinical setting, where the availability of the tissue of interest cannot be exactly planned, this may add a further disadvantage.

However, the major advantages of the FACS-sorting are that the EC fraction can be labeled by multiple antibodies in parallel. Thus, a stringent gating strategy can be employed which will ensure a high purity. Based on experience, no re-sorting of the EC fraction was required, in contrast to the previous MACS-based protocol where multiple rounds of selection had to be employed. This led to a significantly reduced cultivation time until purity from six weeks (MACS approach) to three weeks in the FACS approach, resulting in an improved cell viability enabling a prolonged time window of analysis. Finally, using the FACS-sorting-based strategy, an improvement of the overall isolation success rate could be achieved (increase of 12.5\%). In conclusion, the FACS-sorting based strategy employing 3 antibodies in parallel after tissue digest by combined mechanical and enzymatic tissue digestion had numerous advantages that established this protocol as the method of choice for the isolation of TECs and NECs from human colorectal carcinoma and colon.

\section{Disclosures}

The authors declare that they have no competing interests.

\section{Acknowledgments}

We thank Christian Flierl, Katja Petter, Christina Schnürer (all Division of Molecular and Experimental Surgery), Uwe Appelt, Michael Mroz (Core Unit FACS-Sorting) and Simon Völkl (Core Unit FACS-Immunomonitoring) for excellent technical assistance. This work was supported by grants to EN/MS of the Interdisciplinary Center for Clinical Research (IZKF) of the University Medical Center Erlangen, the German Research Foundation (DFG: FOR 2438, SP2) and the Lutz-Stiftung, by a grant of the Robert-Pfleger-Stiftung to VSS as well as by grants awarded to MS from the German Research Foundation [DFG: KFO257 (sub-project 4), SFB 796 (sub-project B9)].

\section{References}

1. Carmeliet, P. Angiogenesis in health and disease. Nat Med. 9 (6), 653-660 (2003)

2. Carmeliet, P. Angiogenesis in life, disease and medicine. Nature. 438 (7070), 932-936 (2005).

3. Carmeliet, P., \& Jain, R. K. Angiogenesis in cancer and other diseases. Nature. 407 (6801), 249-257 (2000).

4. McDonald, D. M., \& Choyke, P. L. Imaging of angiogenesis: from microscope to clinic. Nat Med. 9 (6), $713-725$ (2003).

5. Hurwitz, H. et al. Bevacizumab plus irinotecan, fluorouracil, and leucovorin for metastatic colorectal cancer. N Engl J Med. 350 (23), 2335-2342 (2004)

6. Naschberger, E., Schellerer, V. S., Rau, T. T., Croner, R. S., \& Stürzl, M. Isolation of endothelial cells from human tumors. Methods Mol Biol. 731 209-218 (2011)

7. Schellerer, V. S. et al. Endothelial cells of human colorectal cancer and healthy colon reveal phenotypic differences in culture. Lab Invest. 87 (11), 1159-1170 (2007).

8. Naschberger, E. et al. Matricellular protein SPARCL1 regulates tumor microenvironment-dependent endothelial cell heterogeneity in colorectal carcinoma. J Clin Invest. 126 (11), 4187-4204 (2016).

9. St Croix, B. et al. Genes expressed in human tumor endothelium. Science. 289 (5482), 1197-1202 (2000).

10. Christian, S. et al. Endosialin (Tem1) is a marker of tumor-associated myofibroblasts and tumor vessel-associated mural cells. Am J Pathol. 172 (2), 486-494 (2008)

11. Grange, C. et al. Isolation and characterization of human breast tumor-derived endothelial cells. Oncol Rep. 15 (2), $381-386$ (2006).

12. Miebach, S. et al. Isolation and culture of microvascular endothelial cells from gliomas of different WHO grades. J Neurooncol. 76 (1), $39-48$ (2006).

13. Richard, L., Velasco, P., \& Detmar, M. A simple immunomagnetic protocol for the selective isolation and long-term culture of human dermal microvascular endothelial cells. Exp Cell Res. 240 (1), 1-6 (1998).

14. Nourse, M. B. et al. VEGF induces differentiation of functional endothelium from human embryonic stem cells: implications for tissue engineering. Arterioscler Thromb Vasc Biol. 30 (1), 80-89 (2010).

15. Sato, T. et al. Single Lgr5 stem cells build crypt-villus structures in vitro without a mesenchymal niche. Nature. 459 (7244), $262-265$ (2009). 